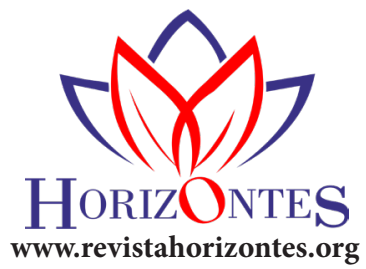

\title{
Literatura de la etnia kichwa de Ecuador
}

\author{
Literature of the kichwa ethnic of Ecuador \\ Literatura da étnica kichwa do Equador
}

\section{Cristóbal Lorenzo Quishpe Lema}

cristobalquishpe48@gmail.com

https://orcid.org/0000-0001-7029-7397

Casa de la Cultura “Benjamín Carrión” Núcleo de Cotopaxi-Ecuador

RESUMEN

En las comunidades ancestrales kichwa de Ecuador, su rica etnoliteratura, hasta la actualidad, casi se ha limitado a la oralidad, en razón de la poca preocupación de la educación aparece como irremediablemente escindida entre dos prácticas por lo común independientes: la escritura y la oralidad. Esta investigación tuvo como objetivo analizar lo que pasa con la literatura en las comunidades ancestrales kichwas de Ecuador y la escasa producción escrita, pues aparece como irremediablemente escindida entre dos prácticas por lo común independientes: la escritura y la oralidad. El estudio se desarrolló bajo el paradigma cualitativo-interpretativo. Los resultados develaron que la geoliteratura y etnoliteratura tienen como propósito el estudio y el análisis de la evolución de la expresión lingüística y etnoliteraria a través del tiempo y espacio. Se concluyó que es necesario acentuar el hecho de que los pueblos ancestrales kichwa de Ecuador están comprometidos a una descolonización no solamente desde los sectores socio-políticos, sino también artístico-literarios.

Palabras clave: Literatura; Etnoliteratura; Geoliteratura; Lingüística; Cultura

ABSTRACT

RESUMO

In the ancestral Kichwa communities of Ecuador, their rich ethno-literature, to date, has almost been limited to orality, due to the lack of concern for education, it appears as irremediably divided between two generally independent practices: writing and orality. The objective of this research was to analyze what happens to literature in the ancestral Kichwa communities of Ecuador and the scarce written production appears to be irretrievably split between two generally independent practices: writing and orality. The study was developed under the qualitativeinterpretive paradigm. The results revealed that geoliterature and ethnoliterature have as their purpose the study and analysis of the evolution of linguistic and ethno-literary expression through time and space. It was concluded that it is necessary to emphasize the fact that the ancestral Kichwa peoples of Ecuador are committed to decolonization not only from the socio-political sectors, but also from the artistic-literary sectors.

Key words: Literature; Ethnoliteratura; Geoliterature; Linguistics; Culture
Nas comunidades ancestrais Kichwa do Equador, sua rica etno-literatura, até agora, quase se limitou à oralidade, devido à pouca preocupação com a educação, aparece irremediavelmente dividida entre duas práticas geralmente independentes: a escrita e a oralidade. O objetivo desta pesquisa foi analisar o que acontece com a literatura nas comunidades ancestrais Kichwa do Equador e a escassa produção escrita parece estar irremediavelmente dividida entre duas práticas geralmente independentes: a escrita e a oralidade. $\mathrm{O}$ estudo foi desenvolvido sob o paradigma qualitativo-interpretativo. Os resultados revelaram que a geolocalização e a etnoliteratura têm como objetivo estudar e analisar a evolução da expressão linguística e etno-literária ao longo do tempo e do espaço. Concluiu-se que é necessário ressaltar o fato de que os ancestrais povos Kichwa do Equador estão comprometidos com a descolonização não só dos setores sociopolíticos, mas também artísticoliterários.

Palavras-chave:

Ethnoliteratura; Linguística; Cultura
Literatura; Geoliterature; 


\section{INTRODUCCIÓN}

En el marco de generar nuevas posturas críticas sobre la importancia del estudio de la relación cultura y lenguaje dentro de la educación, es preciso sustentar en estas primeras líneas que la Etnoliteratura, es decir, las expresiones literarias de las comunidades ancestrales es escasa en el ámbito escolar. Obras como Huasipungo, escrita por Jorge Icaza en el año 1934, la cual ha sido valorada como la representación de la novela social indigenista de la literatura ecuatoriana de carácter serrano; y por su parte el texto Cumandá, de Juan León Mera en el año de 1877, que versa sobre la vida de la selva del Oriente ecuatoriano, lastimosamente son desconocida por la juventud actual.

La mayor parte de la producción literaria de los pueblos ancestrales está solamente en la memoria y expresada oralmente. En tal sentido, por siglos se aferra a sobrevivir. La memoria literaria ancestral está presente en la geoliteratura, como señala Pillet (2017) en su libro: Geoliteratura. Paisaje literario y turismo. El autor, en su escrito, expresa:

Junto a la aportación literaria, no podemos olvidar otras fuentes, aunque ahora no las utilicemos, como es el caso de la pintura, los dibujos, la cartografía, las ilustraciones y la fotografía que ayudan a comprender la idea del paisaje urbano. Se ha afirmado que la literatura, el grabado y la pintura contribuyen a definir como "culturales" paisajes urbanos que sobresalen por su emplazamiento, entorno, entramado y construcciones. (Pillet, 2017, p. 49).

De lo anterior se deriva que la etnoliteratura, como parte de la Antropología socio-cultural, hace referencia a la literatura de las comunidades ancestrales, a los discursos culturales en todas sus formas. Por tanto, surge como proyecto de reconocimiento de la literatura de los que oralmente pueden manifestar las figuras ideológicas, sociales, culturales y religiosas, luego, plasman por escrito en la literatura de los pueblos originarios. Esta literatura otorga un marco de referencia a la realidad socio-cultural. La Etnoliteratura hace reseña a lo imaginario, y a lo que se quiere, y como esto se proyecta en la conducta humana real, por ello es competente para el antropólogo.

De este modo, siguiendo el estudio Ortega (2009) la etnoliteratura posibilita indagar el rol que juega la fantasía en todos los aspectos literarios que constituye las formas a través de las cuales los seres humanos expresan, buscando un vaivén entre observación y emoción, emoción. Esta no es más que participación vital, apertura frente al otro, simbiosis o comprensión del yo a partir de él, comprensión del yo a partir de las diferencias. Al ser la palabra un factor importante dentro del pensamiento y desarrollo del hombre por medio de ella, se llega a conocer al otro cómo es y cómo se desenvuelve y, es la palabra la que ayuda a conocer el origen de la cultura que sea. La palabra muestra la literatura en la etnia; pero también la etnia en la literatura. La etnia entendida como un conjunto de personas que pertenecen a una misma "familia" y generalmente a una misma comunidad lingüística y cultural.

Considerando la importancia de posturas alternas sobre el tema y relevancia actual educativa de la etnoliteratura, se destaca lo expresado por Moyano y Álvarez (2018), quienes explican, desde una perspectiva intercultural, el impacto de los estudios teóricos-interpretativos en la explicación de las particularidades de las voces y expresiones propias de las culturas originarias de América, su potencialidad, belleza y su representación discursiva. En otras palabras son investigaciones que aportan relecturas de las cosmovisiones 
colectivas y prácticas del imaginario estético, las cuales configuran significados en cada pueblo particular.

Quishpe (2018), en su tesis: "La Etnoliteratura como herramienta pedagógica en la enseñanza de la lectura comprensiva en los educandos de quinto año de educación general básica del Centro Educativo Intercultural Bilingüe "Muyu Kawsay", de la parroquia Guamaní, del cantón Quito, Ecuador (período 2017-2018)", dice:

Los discursos literarios orales o escritos, incluso los gestos (los ritos) en la medida que son productos de los imaginarios sociales, constituyen espacios de escenificación de la vida, de las historias sociales, particulares, regionales o simplemente no dichas por la historia oficial por que ha sido totalizadora, homogenizante y universalista sin permitir que los pueblos oprimidos alcen su palabra y compartan la misma (p. 40).

Además, cabe indicar que la Etnoliteratura no es expresada solamente por varones, también hay inspirada por mujeres, especialmente por mujeres de edad adulta y de experiencia que cantan al ser amado, que exclaman a las divinidades, que cuentan a su hijas y nietas para que sepan lo que es la vida. Esto se encuentra cuando hay matrimonios, cantan y loan el "Mashalla Kachunlla" (Solo yerno y solo nuera) la madre de la novia da consejos para la hija y para el yerno, en base de loa.

Esta investigación analiza la literatura en las comunidades ancestrales kichwas de Ecuador, su delicadoestilo, que siempre se ha limitado a la oralidad, y que, por la poca preocupación de la educación, existe insuficiente producción escrita. Por lo cual, aparece como irremediablemente escindida entre dos prácticas: la escritura y la oralidad.
La práctica escritural la cual vino de la cultura occidental exige que se realice dentro de las prácticas occidentales, se sabe que la expresión de sentimientos, lengua y cultura es distinta a la cultura occidental en contraste con la ancestral kichwa.

Así es como, el conjunto de prácticas orales de arraigo local, casi siempre difíciles de disociar de su contexto artístico (música, danza) y social (ceremonia), tiene su tronco central en las narraciones que realizan personas que van de los 70 y más años, es ellos los que enseñan a sus nietos y ha sido imposible olvidar. Ruth Moya en su libro "Taruka, La venada, literatura oral kichwa”, indica: "La literatura oral cumple con una función pedagógica: sus cantores, narradores y poetas actuales han heredado de los viejos amautas la posibilidad de transmitir los hechos legendarios e históricos, las doctrinas morales y estéticas." (Moya y Jara, 2009, p. 13).

Es cierto que para un letrado de la cultura occidental considera como una expresión más arcaica a la expresión verbal humana que evoluciona inexorablemente hacia formas cada vez más sofisticadas de la escritura. La cultura oral ancestral, en pocas palabras, figura incompatible con la modernidad. Con poemas, coplas, cantos, cuentos, acertijos y creencias encarna el milagro de hablar en tres tiempos: primero los sentimientos, luego a los sentidos, finalmente a la inteligencia.

Por consiguiente, grandes poetas de origen occidental y ancestral han dejado escrito sus sentimientos. Tal como se da en las novelas Huasipungo y Cumandá, antes mencionadas. Sin embargo, otros anónimos que tan solo han dejado sus obras en palabras, de ellos y a ellos quizá no recuerdan, sabiendo que en su literatura se encuentra la cosmovisión y filosofía de vida: el Sumak Kawsay. 
La literatura oral kichwa de Ecuador, escasamente está publicada en textos de educación y de divulgación literaria, como se dijo antes, más se conserva en la memoria de la colectividad ancestral. A raíz de la Educación Intercultural Bilingüe en Ecuador, existen investigadores que han documentado en libros, pero, se requiere más investigación para que las expresiones de cantos, poemas, cuentos, anécdotas, dichos, creencias se divulguen por escrito y se ponga en manos de los docentes de Lengua y Literatura y realicen estudios de sus respectivas expresiones en las aulas de la EGB, Bachillerato y Universidad.

\section{MÉTODO}

La investigación se enmarcó en el paradigma cualitativo, bajo un enfoque interpretativo. Es un estudio de orden reflexivo que valora la trascendencia aborigen kichwa en la formación literaria por medio de los diálogos, cantos, poemas, cuentos, anécdotas, dichos y exteriorización de creencias.

\section{Limitaciones del trabajo}

Para sustentar el tema de investigación se realizó revisión bibliográfica visitando bibliotecas y consultando en internet. En las fuentes electrónicas se encontraron varios temas con respecto a la Etnoliteratura aplicada a la educación. En Ecuador hay muy poca experiencia al respecto, por tanto, hay escasa fuente bibliográfica.

\section{DESARROLLO Y DISCUSIÓN}

\section{Reflexión}

Es oportuno, siguiendo el objetivo del estudio, analizar el ayer para valorar el hoy. A Ecuador arribaron los Incas que hablaban el Runashimi, conocido como Kichwa. Además, a los inkas les acompañaron los aimaras, y de esa lengua le han puesto el nombre al volcán más alto del mundo como Cotopaxi, interpretado al castellano quiere decir: "cuello de luna".

Los Inkas a su llegada por el lado sur de Ecuador, ya se encontraron con poblaciones ancestrales que vivían en estas encantadoras tierras. Ellos también ya tenían su propia lengua. Actualmente está desaparecida o existen en topónimos, zoónimos o fitonimios.

Es preciso destacar que, en el estudio, no se utilizó el epíteto "indio ni indígena” para referirse a los habitantes ancestrales, por la siguiente razón: La idea de "indio" fue, sobre todo, una iniciativa de los españoles, es decir, una nominación genérica para los habitantes de Apya Yala que antes y siempre habían vivido en el continente que hoy es América. El concepto "indio" no provenía del sujeto mismo a quien se aplicaba sino de la sociedad que lo conquistó. Colón, luego de su primer viaje, pensó que había llegado a la India Oriental, porque esa era su meta. A la vuelta a España, llevó consigo a personas de lo que hoy es Centro América y, en España les presentó como "indios" de las tierras descubiertas. Para los europeos el "indio" era un ser negado como ser humano, inclusive los sacerdotes de ese entonces, dijeron que no tienen "alma". Para los españoles era el "otro" que lo negaron siempre en su estatus social, le situaron a parte y el trato era como a seres indignos.

La historia de los pueblos ancestrales comenzó a escribirse con la negación de sus culturas mediante la imposición de la lengua castellana y con los signos alfabéticos imperantes, lo peor fue cuando escribieron las lenguas ancestrales con el alfabeto castellano, fue allí en donde se trastrocó toda la riqueza cultural y lingüística de las lenguas originarias. Es más, la escritura de los pueblos ancestrales no fue fonética, sino ideográfica, es 
decir, se trataba de una representación de ideas o palabras a través de imágenes o símbolos, ejemplo, los Kipus.

Las comunidades ancestrales en América del Sur en especial de lo que hoy es Ecuador, si sabían hablar, quiere decir que deben haber tenidos expresiones como: (arawikuna) poemas, (rimaytakikuna) coplas, (takikuna) cantos, (atinamanta hayllikuna) himnos de victoria, (ñawparimaykuna) cuentos, (watuchinakuna) acertijos, (iñinakuna) creencias, estas expresiones lo ponía en juego para las fiestas, para las labores agrícolas, reuniones sociales y para la educación, además, para demostrar un corazón estremecido por el jolgorio o por el dolor, por la esperanza, por el nacimiento de un niño o niña, la nostalgia, por la siembra o la cosecha.

En un recorrido por muchos años por las diferentes poblaciones ancestrales kichwas del Ecuador, se puede notar que hay tres generaciones de autores en la producción de Literatura ancestral. A la primera le tocó abrir camino y se conformó de alfabetizadores (no eran profesores con título), profesores rurales, promotores culturales $y$ supervisores de Educación Intercultural Bilingüe, a la segunda generación, en la mayoría eran los alumnos de la primera generación y abrieron paso a una tercera generación que tiene a su alcance las redes sociales y virtuales para la difusión de sus obras y de su lengua, pero, es escasa. Ahora, muchos jóvenes de comunidades ancestrales del país, tienen vergüenza de hablar en kichwa o decir su lugar de origen, niegan a su propia cultura y a su identidad.

En el diario vivir, siempre se escuchó de boca de los abuelos, de los padres y de los moradores, contar cuentos, leyendas, cantos a las montañas, a los ríos, a los animales, a los espíritus, a la naturaleza misma. Cada mañana, al momento de desayunar siempre los abuelos conversaban lo que habíamos soñado y predecían lo que puede suceder. Por la tarde, de la misma manera, mientras preparaban la merienda, comentaban lo que habían visto o trabajado durante el día y, terminaban contando cuentos, cantando a la mujer, al amor, a la migración de las personas, sueños, mitología y aspiraciones. Todo eso, fue motivo para investigar los cuentos, cantos, tradiciones, fábulas, de vida cotidianas de la gente, esa estimulación fue por las visitas continuas a las comunidades kichwas del país, comunidades enteramente ancestrales algunos de mitimaes traídos del Alto Perú como los Salasaca, Saraguro y Poalos.

Dentro de la Sierra ecuatoriana existen cerros, nevados, laguna, quebradas, vertientes que los moradores hacen alusión en sus conversaciones, así como también de los animales que viven junto al hombre y de los animales silvestres. En esta geografía el habitante tiene en su memoria: cuentos, leyendas, tradiciones cuentos de las lagunas, de los volcanes, de los ríos, cerros y valles.

Los relatos que la mayor parte de persona guardan en sus memorias y es de tradición oral, exteriorizan diferentes historias de acuerdo al lugar, los personajes son salidos de la naturaleza y seres fantásticos que interactúan con los hombres y las mujeres, niños, jóvenes, adultos y ancianos. El universo de relatos que conforman y expresan es una manera de entender el mundo que proviene de tiempos muy antiguos, cuando no existían explicaciones científicas para muchos fenómenos que afectaban a las personas o a la naturaleza. Son pensamientos y expresiones culturales que en la actualidad también pueden contribuir a la recuperación del espíritu originario y de la población mestiza del Ecuador, claro que actualmente tan alejada de sus raíces. Sin embargo, esas raíces son necesarias escarbarlas.

Como resultado de la investigación, se expone un fragmento del canto del matrimonio que se realiza en Maca grande, Maca chico y Pilligsillí del cantón Latacunga, provincia de Cotopaxi. 


\section{Mashalla kachunlla sawaripi taki}

\section{Canto en el matrimonio a la hija y al yerno}

\author{
Ushushita kamachina \\ Tukuy tutamantapi, \\ uktami hatarina kanki, \\ tullpata rikunkapak, \\ kusamanmi mikuchina kanki.

\section{Mashata kamachina} \\ Amuklla warmishinami, \\ payka pantanka, \\ mishki rimaywan, \\ pushana kanki.
}

\author{
Consejo a la hija \\ En todas las mañanas \\ tendrás que levantar rápidamente, \\ para mirar el fogón, \\ tendrás que dar de comer a tu esposo.
Consejo al yerno
Como una mujer humilde, ella se equivocará, y con dulces palabras, la tienes que sobrellevar.

Y como los seres humanos no son eternos, también hay el canto a los muertos que despiden de su Pachamama y Allpamama. Fragmento:

\section{Canto a los muertos}

Adiós ñukapa wasilla, adiós ñukapa kanchalla,

Adiós ñukapa allpa mamakulla, adiós ñukapa tiyaykulla,

Adiós sumak kuyllurwa, adiós sumak puyulla.
Adiós mi casita, adiós mi patiecito.

Adiós mi madre tierra, adiós mis banquitos.

Adiós mi hermoso lucerito, adiós hermosa neblinita.
La literatura oral en lengua kichwa de Ecuador tiene, por otro lado, un sesgo ritual porque magnifica aquellas instancias del trabajo y de la vida, instancias en las cuales se materializa su ser espiritual. La oralidad constituye un instrumento insustituible, pues, tiene en la memoria de un individuo y de una colectividad, la base cultural de una sociedad más allá de los soportes materiales se conserva en la memoria. Goody (1996) dice: “... en cada generación, por lo tanto, el recuerdo individual medirá en la herencia cultural de tal manera que sus componentes se ajustaran a los viejos a través del proceso de interpretación".

En las comunidades ancestrales, la literatura oral cumple con una función pedagógica, sus cantores, narradores y poetas actuales han heredado de los viejos "amautas" (sabios) la posibilidad de transmitir los hechos legendarios e historias, las doctrinas morales y estéticas. 
Con los poemas, coplas, cantos, cuentos, acertijos y creencias, inicialmente emociona, conmueve y los estremece a niños, jóvenes y ancianos. Hace gozar y sufrir, luego, la memoria sensorial lleva a la imaginación, a sentir sabores, olores. Las palabras como si fueran notas musicales diestramente elaboradas comparten para el deleite de otras personas.

Por otro lado, en el libro Elementos Básicos para la Gestión Educativa expresa: "Si Jesús enseñó en base de parábolas, los kichwas, especialmente a los niños y niñas, les enseñamos en base de los cuentos". El objetivo, se quiere tener un pueblo feliz que practique la ética y la moral." (Quishpe, L. 2019, y Quishpe, 2016).

La literatura ancestral no es fría, que siempre termina en tragedia, esta Literatura tiene algo de picaresca y de sabiduría, es para formar al hombre nuevo para que viva feliz. Ver el siguiente fragmento del cuento.

\section{Ullawanka waywalliwan rimaymanta nawparimay - Cuento del diálogo entre el gallinazo con la garza}

\section{Ullawanka ñwparimay}

Pishkukunaka chikan chikanmi kan, wakinka hatunmi, wakinka uchillami kan, ullawanka paypa patpaka yapa yanami kan, ranti waywallipa patpaka yapa yurakmi kan.
Ullawankaka patpapa waywallimanta chikihushkami kashaka; chaymanta shuk punchaka mishkishimiwanmi kayashka.

-Mashi waywalliku imanallatak kapanki, imatatak rurahunkika, sapallachu purikunki-, nishkashi. Waywallika: -Ari mashi ullawanka, ñukaka sapallami kapani, mikunata mashkashpami kapani-, kutichishka. Waywallipash ullawankata tapushkami, -Ullawankaku, kanka imatatak rurakupanki, kanpa warmitaka maypitak sakiparkanki-. Ullawankaka: -Ñukapash mikunata mashkashpami purikunika, ñukapa warmika ñukanchikpa wawakunawanmi sakirirka-, kutichirka. Ullawankaka: -Mashi waywalliku kanka imamantatak yurakmi kapanki-, tapurka. Waywalika: -Ari, ñukapa patpaka ruyaktami charipani, tukuy punchami kuchaman rini, chaypi armarini, chaymanta nukapa patpaka yurakmi kan, mashiku-, nispami kutichirka

\section{Cuento del diálogo entre el gallinazo con la garza}

Las aves son diferentes, algunas son grandes, en cambio otras son pequeñas, las plumas del gallinazo son bien negras, en cambio las plumas de la garza son bien blancas.

El gallinazo era envidioso de las plumas de la garza, por lo tanto, un día le llamó con lisonjas.

El gallinazo le había preguntado: - ¿Amiga garcita cómo estás?, ¿qué estás haciendo?, ¿estás caminando solitaria? -. La garza le había contestado: -Si amigo gallinazo, estoy solitaria, estoy buscando comidaDel mismo modo la garza le había pregunta al gallinazo: - ¿Gallinacito, tú qué estás haciendo?, ¿en dónde dejaste a tu esposa? -. El gallinazo le había dicho: -Yo también estoy buscando comida pues, mi esposa se quedó con nuestros hijos. El gallinazo le averiguó: - ¿Amiga garcita porqué eres tan blanca? La garza le había contestado: -Si, yo tengo las plumas blancas, porque todos los días voy a la laguna, allí me baño, por eso mis las plumas son blancas, amiguito-. 
Rikupay waywalliku, ñukapa patpaka yapa yanami kan, ñukapash patpatata yurakmi charina nini, mashiku-, nishkashi. Waywallika: -Shina kashpaka, tukuy puncha tukuy tutami, kuchapi yallina kanki, kanpa patpa yurakyakunkama-, nishkashi.

Shinaka, waywallika ullawankataka kuchaman aparka, chaypi, waywallika nirka: -Ullawankaku, kaypimi, tukuy puncha, tukuy tuta armashpami yallina kanki, kanpa patpata yurakyachun. Chay hawamanta ñukaka tapushpa kaparisha. -Mashiku ña kanpa patpataka yurakchu kan, nishpa-. Ullawankaka: -Ari mashikuku shinami rurak rinchik-, nishpami kutichirka. Ña chishi kakpika, waywallika hawamanta, - ¡Mashiku ña kanpa patpaka ña yurakchu kan! -, tapurka, ullawankaka: -Manarak, manarak, nishpami kutichirka. Shinami kimsa punchami yallirka, ña kipaka ña mana kutichirka, waywallika, imatak yallin, payta rikusha nishpa, kuchaman uriakurka, ullawankaka wañushka wampushpami karka.

\section{YUYAY:}

Uyapaychik mashikuna, ñukapa kikin ukkutaka chayshina kan, shinami wañuk rinchik, mana kutishuk shina mashkak rinchik, chaymanta kuyana kanchik.

En un pueblo que se practica el genocidio, donde hay hegemonía de uno de los grupos y, como consecuencia de ello una diglosia, es muy difícil hablar de interculturalidad literaria. Personas que se creen "cultas" han manifestado que reconocer a las lenguas y a las culturas ancestrales es retroceder, volver al pasado y permanecer en la ignorancia.
-Míra garcita, mis plumas son tan negras, yo también quiero tener las plumas blancas-.

La garza le había aconsejado: -Entonces tiene que pasar en la laguna todo el día y toda la noche, hasta que las plumas estén blancas.

Entonces, la garza le había llevado al gallinazo a la laguna, allí le había dicho: -Gallinacito aquí tienes que pasar bañándote todo el día y toda la noche, hasta que tus plumas estén blancas. -Desde arriba te he de gritar preguntando-. - ¿Amiguito tus plumas ya están blancas? -. El gallinazo había aceptado la propuesta: -Si amiguito así haremos-. Ya al atardecer la garza le gritó preguntando: - ¡¿Amiguito tus plumas ya están blancas?! El gallinazo le había contestado: -Todavía no, todavía no-. De este modo ya habían pasado tres días, luego ya no le contestó, la garza se preguntó: ¿Qué pasó?, voy a ver, bajó a la laguna, el gallinazo había estado flotando muerto.

\section{MORALEJA:}

Por favor razonen amigos, nuestro cuerpo así es, así hemos de morir, no busquemos otra forma de ser, por tal razón debemos tener estimando.

Sin embargo, se puede decir que el pueblo ancestral por medio de los cuentos les forma en valores a sus hijos. Por ejemplo, para que los jóvenes y niños no sean mentirosos, se contempla el siguiente cuento: 


\section{Llulla wamramanta ñawparimay- Cuento del muchacho mentiroso}

Kishwarmallki ayllullaktapika shuk michik wamrami tiyashka. Payka tukuy puncha urkumanmi llamakunata, kuchikunata, chitakunata, wakrakunata, ushukunatapash michinkapakmi rik kashka. Payka allkutin rik kashkachu, mana sapallachu michik kashka.

Chay wamraka yapa llullapachami kashka, ninkuna. Shuk puncha ña urkupimi michishpa kashpaka, payka kaytami yuyashka, kunanka tukuy Kishuarmallki ayllullaktamanta runakunata kushparichik rini.

Wamraka shinchimi kaparishka. ¡Atuuukmiii shaaamuuun!, aayllukuuunaaa ¡Atuuukmiii shaaamuuun! Payllatak asikushkami.

Shina kaparikpimi tukuy Kishwarmallki ayllullaktamanta runakunaka, millaysiki atuk llamakunata mikukun, nishpa, tantanakushkami, utkami kaspikunawan urkuman rishkakuna, ña chayman chayakpika, mana ima rikurishkami, runakunaka tikrashkakuna. Wamraka wiksata hapishpa payllatak asikushkami.

Llulla wamraka, kutinmi kaparinata yuyashka, shinaka shinallatak: - ¡Atuuukmiii shaaamuuun!, aaayllukuuunaaa ¡Atuuukmiii shaaamuuun! kaparishka. Chay shina kaparikpika, kutinllatak tukuy Kishwarmallki ayllullaktamanta runakunaka tantachishkallami urkuman rishka. Chayman chayashpaka mana ima atukta rikurishkachu, ninkuna. Kutinmi wamraka wiksata hapishpa payllatak asikushka.

Ña chawpi puchaka, llullasiki wamraka chimpapi hatun manchanay atuktami rikushka, payka kutin kaparikpika Kishwarmallki ayllullaktamanta runakunaka mana uyashkakunachu, chay wamraka llullami kan, nishpa, mana pi runakunaka tantanakushkachu. Chaykama atukka urkupika shuk sumak llamakuta hapishpa mikunkapak apashkami, ninkuna, wamraka chayta rikushpa achkatami wakashka. -Kunanka ñukapa yayaka imatak ninka-, nishpa.

YUYAY: Chaymanta mana yankata llullanachu kanchik, mutsushkapi mana pi mana yanapankachu.

\section{Cuento del muchacho mentiroso}

En la comunidad de Kishwarmallki había un chiquillo pastor. Todos los días acostumbraba ir a pastar borregos, chanchos, chivos, vacas y burros en el cerro. Él iba acompañado de perro, el no pastaba solito.

Comentan que aquel chiquillo era un contumaz mentiroso. Un día que estaba pastando, él había pensado lo siguiente: Ahora voy a levantar a todas las familias de Kishwarmallki.

El chiquillo gritó fuertemente. ¡El lobo vieeeneee!, famiiiiliaasss ¡El lobo vieeeneee! Entre él estaba riéndose.

$\mathrm{Al}$ oír los gritos las familias de la comunidad de Kishwarmallki, conversaban que el furibundo lobo está comiendo los borregos, se reunieron y rápidamente se fueron al cerro con palos, ya estando allí, no vieron nada, entonces procedieron a regresar. El chiquillo solito se reía cogiéndose la barriga.

El chiquillo mentiroso, otra vez pensó gritar, entonces de la misma manera gritó: - ¡El lobo vieeeneee!, famiiiiliaasss ¡El lobo vieeeneee! -. Luego, nuevamente todas las familias de Kishwarmallki reuniéndosen se fueron al cerro. Al llegar allá, manifiestan que no vieron a ningún lobo. Otra vez el chiquillo solito se carcajeó cogiéndose la barriga.

Ya al medio día, el chiquillo mentiroso vio al frente a un espantoso lobo, él nuevamente gritó a las familias de Kishwarmallki, ellos no le hicieron caso, entre ellos comentaban que ese chiquillo es mentiroso, nadie de las personas no se reunió. Dicen que hasta eso el lobo en el cerro se llevó para comer a un lindo borreguito, al ver eso el chiquillo lloró amargamente. Decía: -Ahora qué dirá mi papá-.

MORALEJA: En tal razón, por ningún motivo debemos mentir, cuando necesitemos, nadie nos hará caso ni nos ayudarán 
Entre las sociedades ancestrales no existen problemas en cuanto al reconocimiento y el respeto de la lengua y la cultura. La ausencia del trato intercultural se da en la sociedad hegemónica (supremacía que un estado o un pueblo ejerce sobre otro), en cambio, la interculturalidad y comunicación entre comunidades ancestrales es un hecho.

Por consiguiente, para que exista la interculturalidad en la literatura es necesario que una sociedad donde existen pueblos de distinta lengua y cultura aprendan y conozcan sistemáticamente las lenguas y culturas existentes en el país, solo así, en lo sucesivo no habrá problemas de expresarse ya sea en lengua ancestral o en castellano y actuar según los rasgos culturales respectivos en cualquier situación que se encuentren los individuos.

Dentro de la literatura ancestral se ha encontrado que en la parte zoológica hay cuentos fábulas, leyendas, dichos del conejo, venado, lobo, zorro, oso, gallinazo, caracol, zarigüeya, lechuza, oveja, llamingo, ratones. De la misma manera, en lo que corresponde a los espíritus están los duendes, el cerro, el arco iris, arco blanco, sacha runa, la viuda, la caja ronca.

Los habitantes de las comunidades kichwa de Ecuador son herederos de una abundante, rica y antigua tradición de cultural oral, que se materializa, por decirlo así, en la oralidad de su Literatura. Este fenómeno es común en las culturas ágrafas, pero con legitimidad única que se puede preguntar el significado de las antiguos memorias.

Se puede ver todavía que el poeta, el narrador, el músico, como otros artistas y sabios del pueblo kichwa (tejedores, ceramistas, curadores, matemáticos...) antes de ejercer su oficio a menudo pasan por un baño ritual en las cascadas o fuentes sagradas. Allí invocan a los poderoso Awkikuna y Apukkuna ancestrales pidiendo la fuerza necesaria para acometer la organización.
La iniciación ritual de los artistas, está precedida por un sentido de delegación o herencia. Es del padre de quien se recibe el encargo de cantar, es quien transmite a su hijo mayor o al mejor, al más sabio, a quien delega, luego, continúa en espiral esa obligación y ese derecho.

En la cultura kichwa, se ha encontrado que la fábula es un andamiaje de carácter expresivo de preferencia para transmitir las versiones pedagógicas de su mundo moral; posiblemente por esa razón dicha área temática la que con mayor flexibilidad ha admitido en préstamo algunas fábulas clásicas. Sin embargo, en la medida en que se transmiten en lengua kichwa entre los propios kichwas para sus propios fines sociales, dejan de ser meros préstamos...

La traducción y la transposición a la forma escrita ha sido una de las empresas más complejas por varias razones. En primer término, los textos compilados proceden de variadas comunidades ancestrales, lo que exigía un conocimiento de los modismos, léxico, etc., de los diferentes dialectos kichwas. Por otro lado, rescatar el espíritu y la trascendencia del pueblo kichwa, su único autor, parecía rebasar las limitaciones personales.

Con respecto a la primera dificultad, es decir, la transposición de las formas orales del dialecto local, a una forma escrita, se puede guiar por el criterio impulsado por todas las organizaciones de pueblos ancestrales, esto es, utilizar una grafía unificada y unas mismas reglas de escritura de la lengua kichwa. $\mathrm{Y}$, segundo, la tarea fue más compleja, puesto que se trataba no sólo de buscar el mero equivalente lingüístico sino de transmitir una concepción y una estructura narrativa completamente distinta a lo que estamos acostumbrados, podrían o no podrían respetar los no kichwas. 
Es de suponer que este trabajo de investigación de la Etnoliteratura tiene como destinatario fundamental para el propio pueblo kichwa, si bien los textos en su oralidad y escritos, son un patrimonio colectivo.

La selección poética mínima, cumple la misma función, esto es, esclarecer el contexto en el cual se canta la canción a la cosecha hakchima/hakchiña (palabra aimara: llevar en las manos algo sin soltar), el recoger de los granos tiernos en Pawkar Raymi, en la siembra, el desyerbe y otras actividades. Aquí tenemos el canto a los granos tiernos.

\section{Llullu murukunaku - Granitos tiernos}

Mishki chukllukuta, kushillami hapik rini, yanushka mikunkapak, shinaka, tipishpami apak rini.

Llullu purutukuta, kushillami hapik rini, kachiwanmi mikunkapak, shinaka, ishkushpami yanuk rini.

Kuy kusashkawanka, llullu papakunakuta, utkami akllak rini, shinaka, kushillami llushtik rini.

Llullu murukunawan, yanushka mikunkapak, yurakyamuklla sampukuta, shinaka, kushillami pitichik rini.

\section{CONCLUSIONES}

Con lo que se ha expuesto en este trabajo, se quiere recalcar el hecho dequelos pueblos ancestrales kichwa de Ecuador están comprometidos a una descolonización no solamente desde los sectores socio-políticos, sino también artístico-literarios.

Esto obedece porque frente a la hegemonía de la sociedad occidental, los autores de la Etnoliteratura de origen ancestral tratan de descubrir su propia cultura dentro del terreno discursivo del otro.
Choclito dulce, contento te cogeré, para comer cocinando, siendo así, deshojando te llevaré.

Frejolitos tiernos, contento te cogeré, para comer con sal, siendo así, desgranando cocinaré.

Pues con cuy asado, tiernas papitas, rápido cavaré, siendo así, contento pelaré.

Con granitos tiernos, para comer cocinando, blanquecina calabacita, siendo así, contento te cortaré.

Por otro lado, la heterogeneidad de los escritores de Etnoliteratura les permite justamente recurrir a tradiciones, imaginarios diferentes para producir un discurso original que logra poner de relieve otras lenguas y otros pensamientos. Los últimos trabajos, expresados dentro de la Etnoliteratura, ponen frente a discursos atravesados por tensiones evidentes entre escritura y oralidad.

Así, las maneras diferentes de relacionarse de los escritores de Literatura con diversos seres, 
humanos y no humanos, mediatizada por el lenguaje y la lengua, es lo que se ha denominado filosofía poética, poniendo de relieve que se encuentra hasta en las estructuras profundas de los poemas, la sustancialidad de la palabra.

En tal sentido, las manifestaciones literarias guardadas en la memoria y escritas no sólo ponen ante escritos diferentes, sino que vienen a desafiar al lector occidental y a desafiar la hegemonía discursiva impuesta por la colonia española. En este sentido, la Etnoliteratura contemporánea se inscribe en una descolonización del saber y participa en la construcción de nuevos horizontes discursivos, despojados por las estructuras colonizadoras que mediatizan los conocimientos ancestrales del mundo kichwa es hora que la Etnoliteratura esté presente en el análisis de la educación en general del Ecuador.

De todos modos, las expresiones poéticas del "profetismo" andino u oriental del Ecuador, no es lo mismo, en efecto, "narrar" o "gritar" la revolución cósmico-social. En las narraciones, la expectativa de un Pachakutik, resultan las pautas que ofrece la tradición y no compromete la práctica social de la comunidad "oral", mientras que los cantos (orales) y los poemas (escritos) parecen, más bien, querer acompañar un movimiento "revolucionario" en marcha. Porque dadas las condiciones de marginalidad, de casi clandestinidad de la expresión verbal kichwa, no se sabe hasta qué punto los pocos ejemplos que se adjunta y, se pone a consideración son representativos para inscribir en la educación de los ancestrales y no ancestrales.

En definitiva, los cuentos, como la poesía, el cuento y el canto de la cultura kichwa del Ecuador, dan una idea del mundo simbólico completo, muy ameno y atractivo para los lectores y se espera que en un futuro muy próximo toda la Literatura ancestral o Etnoliteratura se proyecte y esté en manos, a través de espacios de apreciación y debate escolar, de todo el mundo para que sientan la riqueza y armonía de esta encantadora cultura.

REFERENCIAS

Goody, Jack e IAN, Watt. (1996). "Las consecuencias de la cultura escrita", en cultura escrita en sociedades tradicionales, Barcelona: Gedisa

Moya, R. y Jara, F., T. (2009). La venada, Literatura oral kichwa. https://fdocuments.in/document/ taruka-la-venada-literatura-oral-kichwa.html

Moyano, M., y Álvarez, M. E. (2018). Hacia la recuperación de "la voz otra": la etnoliteratura como discursividad y perspectiva. Sociedades de paisajes áridos y semiáridos., 11, 259-274

Ortega, C.L.Z. (2009). El espacio dela etnoliteratura. Mopa Mopa, 1(19), 7-24

Pillet, Capdepon, F. (2017). Geoliteratura, Paisaje literario y turismo, España - La Mancha, SBN: 9788491710646

Quishpe Mendoza, C. A. (2018). La etnoliteratura como herramienta pedagógica en la enseñanza de la lectura comprensiva en los educandos de quinto año de educación general básica del Centro Educativo Intercultural Bilingüe "Muyu Kawsay”, de la parroquia Guamaní, del cantón Quito: UASB

Quishpe Lema, C. (2019). Kichwa shimipa sumak rimay - Literatura de la Lengua Kichwa, Casa de la Cultura "Benjamín Carrión” Núcleo de Cotopaxi. Latacunga Ecuador

Quishpe Lema, C.y Quishpe M, X. (2016). Kichwapa Shimiyuk Kamu, ARCOIRIS Producción e impresión, Quito - Ecuador. UTC 